\title{
Mechanical properties of epoxy/boehmite nanocomposites in dependency of mass fraction and surface modification - An experimental and numerical approach
}

\author{
M. Jux ${ }^{1)}$, J. Fankhänel ${ }^{2)}$, B. Daum ${ }^{2)}$, T. Mahrholz $^{3)}$, M. Sinapius ${ }^{1)}$ and R. Rolfes ${ }^{2)}$ \\ ${ }^{1)}$ Institute of Adaptronics and Function Integration, Technische Universität Braunschweig, Langer Kamp 6, \\ 38106 Braunschweig, Germany \\ ${ }^{2)}$ Institute of Structural Analysis, Leibniz Universität Hannover, Appelstraße 9A, 30167 Hannover, Germany \\ ${ }^{3)}$ Institute of Composite Structures and Adaptive Systems, DLR (German Aerospace Center), Lilienthalplatz 7, \\ 38108 Braunschweig, Germany
}

\begin{abstract}
Boehmite nanoparticles show great potential in improving the matrix dominated mechanical properties of fiber reinforced polymers. For the material design process and the prediction of the nanocomposite's properties, knowledge about the material behavior of the constituent phases and their interactions is crucial. Since the chemical composition of the particle surface can strongly affect the interaction between particle and matrix, the influence of particle surface modification and mass fraction on mechanical properties (tensile modulus, tensile strength, fracture toughness) and failure mechanisms of nanoparticle reinforced epoxy resins is investigated using experimental and numerical methods. In the present work, unmodified and thus chemically reactive boehmite particles are compared to acetic acid modified particles with supposedly lower chemical reactivity and thus worse particle-matrix bonding. A linear relationship between particle mass fraction and tensile modulus/fracture toughness is experimentally found with a maximum increase of $26 \%$ in tensile modulus and $62 \%$ in critical energy release rate for a particle content of $15 \mathrm{wt} \%$. Furthermore, the experiments indicate that the acetic acid surface modification increases the tensile modulus (up to 6 $\%$ compared to the unmodified boehmite particles), but at the same time not significantly affects the tensile strength or the fracture toughness. Molecular Dynamic Finite Element Method (MDFEM) simulations are conducted to identify and understand the mechanisms induced by nanoparticles. The material properties of both, modified and unmodified, nanoparticle systems are discussed in relation to the change of particle-matrix bonding strength and interphase morphology. While simulation results of the unmodified system show an outstanding agreement with the experiments, the acetic acid modified system deviates significantly. In conclusion, it seems that additional effects have to be considered to completely understand the material behavior.
\end{abstract}

Keywords: boehmite, nanoparticles, epoxy resin, surface modification, mechanical properties, numerical methods

\section{Introduction}

Fiber reinforced composites are regarded as one of the most important future lightweight materials. Major weaknesses are their matrixdominated properties. These properties can be improved by adding filler materials. Various studies [1-4] show that in particular nanoscaled fillers, such as nanoparticles and 
nanotubes, possess desirable properties as matrix additives in composite materials. The reinforcement effect depends on their shape, size, content, and particle-polymer interaction. The work of Kango et al. [5] reviews in detail the production of nanocomposites and their mechanical properties with a specific focus on surface modified, inorganic particles.

The incorporation of nanoparticles in polymers can generally improve selected matrix dominated properties of fiber reinforced composites, like shear strength, shear modulus, compressive strength, fracture toughness or impact resistance. For example, Arlt [6] reports that a boehmite particle content of 15 wt\% leads to an increase in these properties up to $25 \%$ for a carbon fiber/Bisphenol-A-diglycidylether composite compared to the reference composite without particle reinforcement. Other studies confirm these results. Uddin \& Sun [7] report a significant increase in compressive strength and transverse tensile strength for silica particle reinforced glass/epoxy composites. Subramaniyan \& Sun [8] obtain similar results for nanoclay/glass/epoxy composites.

In contrast, other studies report opposite results. Shahid et al. [9], alike Arlt [6], investigate boehmite/carbon fiber/epoxy composites with different particle surface modifications, i.e. lysine and parahydroxybenzoate. For small particle weight fractions, tensile and flexural properties improve. Fractions above 5 wt $\%$ show deteriorate properties compared to the unloaded composite. In addition to this, Siddiqui et al. [10] report an ambivalent behavior of nanoclay/epoxy composites, as flexural modulus and fracture toughness increase, while flexural strength and impact toughness decrease. Apparently, the same particle/matrix system affects the mechanical properties differently. In particular, it appears that the surface modification is a decisive factor for the effect of the particle filler on the mechanical properties. Thus, the research on boehmite nanoparticles modified with acetic acid may provide an opportunity to generate further knowledge concerning the effects of modified nanofillers on the mechanical properties of boehmite/epoxy composites.

The comparison of the results of Arlt [6] and Shahid et al. [9] demonstrates that the particle/matrix bonding and the resulting interphase are of vital importance to the mechanical properties of the composite materials. Following the argumentation of Arlt, nanoparticles and their surface modifications strongly affect the morphology of the surrounding matrix by impeding the cross-linking reaction and thus forming a soft interphase around the particles. It is assumed that hydroxyl or other functional groups at the surface of the particle or at the end of a molecule, acting as surface modification, are able to react with epoxy groups of the matrix during the curing reaction. As a result, the particle-matrix interphase becomes softer due to a higher density of particle-matrix bonds. This hypothesis is substantiated by previous numerical atomic force microscopy simulation studies [11]. Generally, the particles can have different effects on the curing behavior of the matrix. Putz et al. [12] systematically varied the cross-link density in an epoxy/carbon nanotube composite to characterize the effect on the formation of interphases. Based on their results, Putz et al. proposed three different mechanisms. The first mechanism is the restriction of the mobility of the polymer chains due to attractive forces between the particle and the matrix. Secondly, the nanoparticles lead to an altered thermal conductivity that changes the curing behavior. The third mechanism is a disruption of the polymer network near the 
particle-matrix interface because of steric limitations or phase segregation.

The present study is an attempt to contribute to the understanding of the effect of surface modifications of nanoscaled matrix additives on the mechanical properties of epoxy/boehmite nanocomposites. Therefore, the mechanical properties of epoxy resins, filled with either unmodified or acetic acid modified boehmite particles, are investigated experimentally and numerically. Unmodified boehmite particles are assumed to chemically interact with the polymer matrix. The hydroxyl groups of the unmodified boehmite might lead to an adsorption or covalent bonding to reactive groups of the matrix. These effects lead to a change in cross-link density in the vicinity of the particles. In contrast, the acetic acid modification, covalently bonded to the boehmite particles utilizing the carboxyl group of the acetic acid molecule, is chemically non-reactive on the opposite molecule end. The chemically nonreactive molecule end is assumed to reduce or prevent particle-matrix bonding, resulting in a lower total surface reactivity of the particles.

By surface reactivity, the authors refer to attractive and repulsive forces affecting the particle-matrix interactions. The surface reactivity and wetting properties of the nanoparticle surface change due to different surface modifications, having an influence on the particle-matrix interphase by changing the morphological properties (degree of cross-linking, chemical composition). It is expected that the macroscopic mechanical properties of the nanocomposites are directly influenced by the surface configuration of the boehmite particles. Regarding the mechanisms stated above, four hypotheses are formulated. The authors refer back to them in Section 5 to discuss their adequacy to explain experimental and numerical results regarding the chemical structure, the mechanical stiffness, the strength at fracture under tensile loading and the fracture toughness.

1. Hypothesis: The acetic acid weakens the particle-matrix bonding and reduces the development of a particle-matrix interphase.

Reasoning: The methyl group of the acetic acid is non-reactive and thus no reaction with the polymer occurs. Depending on the degree of surface loading, the particle-matrix bonding is reduced. This leads to a reduced influence on the epoxy cross-linking and thus to an undisturbed epoxy matrix in the vicinity of the particles (no or only a small interphase region).

2. Hypothesis: Due to the presence of the acetic acid modification, particlematrix bonding is weakened, resulting in lower overall stiffness. Reasoning: A weaker particle-matrix bonding reduces the elastic stiffness. However, a less disturbed epoxy network has the opposite effect. Previous simulations [11] indicate that the influence of reduced bonding is dominating the influence of a less disturbed epoxy network in the interphase. Thus, in total a reduction of the Young's modulus is expected due to acetic acid modification.

3. Hypothesis: The acetic acid reduces the tensile strength.

Reasoning: Weaker particle-matrix bonding caused by acetic acid leads to decreased interfacial stress transfer. In this case, the particle essentially acts as a defect. Thus, there are high local stress concentrations around the particles leading to a decrease in maximum tensile strength. 
4. Hypothesis: Due to the weakened particle-matrix bonding caused by the acetic acid, the particles have a higher tendency to de-bond during the fracture.

Reasoning: The particle induced toughening mechanisms depend on particle size and stiffness and the morphology of the particle-matrix interphase. Crack deflection, particle fracture and crack pinning occur for strong particle-matrix bonding. Weak particle-matrix bonding leads to debonding and subsequently to plastic void growth.

\section{Materials}

\subsection{Epoxy}

The material used in this study is an epoxy resin, diglycidyl ether of bisphenol A (DGEBA) (Araldite LY556; Huntsman), cured with an anhydride curing agent, 4-methyl-1,2cyclohexanedicarboxylic anhydride (Aradur HY917; Huntsman) and accelerated by an amine, 1-methyl-imidazole (DY070; Huntsman). The matrix components are mixed in the proportion of 100:90:1 parts per weight (epoxy:hardener:accelerator).

\subsection{Boehmite}

Boehmite $(\gamma-\mathrm{AlO}(\mathrm{OH}))$ is an oxide-hydroxide mineral. It consists of double layers of oxygen octahedrons bonded to a central aluminum atom. The outfacing oxygen atoms are connected to the hydroxyl groups of the obverse octahedral layer via hydrogen bonds. Boehmite exhibits an orthorhombic unit cell (see Fig. 1a), as reported by Bokhimi et al. [13] and experimentally confirmed through Xray diffraction carried out at the Institute for Particle Technology of TU Braunschweig [14]. In this study, commercially available spray dried boehmite nanoparticles with an orthorhombic shape (see Fig. 1 b) and a primary particle size of $14 \mathrm{~nm}$ are used (DISPERAL HP14, SASOL).

a)
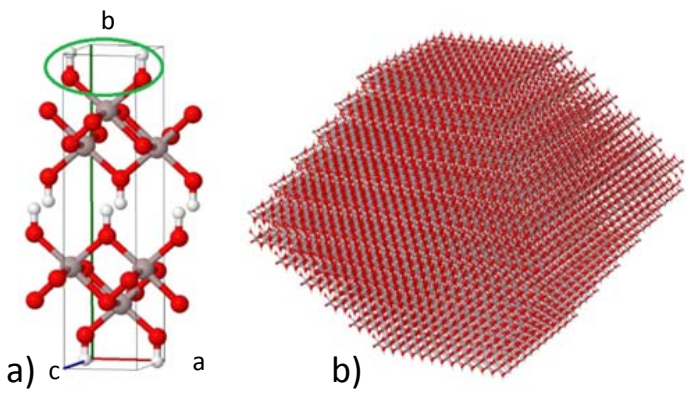

Fig. 1 a) Boehmite $\mathrm{cmcm}$ unit cell ( $a=3.693 \AA, b=12.221$ $\AA$, $c=2.865 \AA$; green mark: surface hydroxyl groups) and b) Orthorhombic shape of the used boehmite primary particles; Pictures of atomic structures created with Jmol [15]

Boehmite behaves mechanically ambivalent with respect to the stiffness caused by the layered structure, as discussed in detail by Fankhänel et al. [16]. Kango et al. [5] state that inorganic nanoparticles are inclined to form agglomerates and thus should be modified to improve the dispersion stability and the compatibility of the particle and the matrix. With decreasing particle size, the ratio of surface area to particle volume and thus the number of surface atoms increases, leading to an increase of surface reactivity and attractive forces between the nanoparticles. Thus, the possibility of particles forming agglomerates increases. The influence of agglomeration on the mechanical properties of epoxy/boehmite composites is summarized in previous studies [17]. The particle-matrix interaction can also be affected by a reactive surface modification producing strong adhesive bonds between particle and matrix, improving the interfacial stress transfer.

2.3 Particle surface modifications and particle-matrix-interphase

Understanding particle-matrix interactions is crucial to comprehend the effect of particle surface modification on the mechanical properties of nanocomposites. Palimi et al. [18] state that the influence of particles on 
mechanical properties is driven by agglomeration and changed cross-linking density as a result of steric hindrance by the particles. Increased mechanical properties due to surface modification of particles are assumed to originate from stronger physical and chemical interactions between particle and matrix. To facilitate stronger particle matrix bonds, surface modifications have to participate in the polymerization processes and covalently bind to at least one of the reactants of the resin system. For nonreactive modifications, the opposite applies. Javidparvar et al. [19] as well concluded that surface modifications can enhance interfacial interactions between particle and matrix through covalent bonding. In the framework of this study two different particle configurations are used for the experiments: unmodified boehmite with innate surface hydroxyl groups and boehmite modified with acetic acid.

\section{Experimental and numerical methods}

\subsection{Specimen preparation}

The nanoparticles are processed in highly viscous suspensions with mass fractions of 30 wt\%. Generally, different types of loading can be used for dispersion, like ultrasonification or shear stressing between surfaces and laminar flow. Highly viscous suspensions require higher stress levels for dispersion. Thus, a kneader was utilized as dispersing device, applying mainly laminar shear flow. The degree of agglomeration after dispersion was verified via dynamic light scattering (DLS) and scanning electron microscope (SEM). The measuring range of the DLS is 0.5 to $10000 \mathrm{~nm}$. For both particle systems, no significant deviations in particle size distribution in the sub-micrometer range were found via DLS. SEM-micrographs showed only rarely larger agglomerates (1-2 $\mu \mathrm{m})$. Detailed information about the dispersion process and the characterization of the degree of agglomeration were previously published [17][14].

The acetic acid content is specified with $1.5 \mathrm{wt} \%$ according to the analysis certificate supplied by the manufacturer (SASOL, Germany). The modification is realized during the manufacturing of the particles in an aqueous phase, subsequent to the synthesis and before drying. It is assumed that at this time, due to high attractive forces between the particles, there are already agglomerates present, and thus, not every primary particle and not the entire surface of all primary particles are modified with acetic acid. However, the exact agglomeration state during the modification process is unknown. Additionally, agglomerates might be even finer redispersed during manufacturing of the specimens, causing an uneven coverage of the agglomerate surface. Thus, a precise determination of the loading ratio of the acetic acid modification is impossible.

The effect of surface modification on the mechanical properties of epoxy/boehmite composites is examined via tension and fracture tests for a constant average particle size of approximately $100 \mathrm{~nm}$ and a particle mass fraction between 0 and $15 \mathrm{wt} \%$. First, highly viscous suspensions with mass fractions of 30 wt $\%$ are blended with DGEBA by using a vacuum centrifugal mixer. To obtain a high quality mixture, it is blended at least 2 times with rotational speeds up to $2100 \mathrm{rpm}$. Simultaneously the mixture is subjected to vacuum (up to $3 \mathrm{mbar}$ ) in order to reduce trapped air. In further blending steps, hardener and accelerator are added. Visual tests after each blending step indicate the quality of the mixture. Prior to further processing the final mixture is filtered with a sieve (mesh size: $190 \mu \mathrm{m}$ ). Subsequently, the mixture is degassed with a desiccator at a vacuum of 0.2 mbar. For the manufacturing of test specimens, the mixture is casted in a 
pre-heated tool $\left(80^{\circ} \mathrm{C}\right)$, treated with a water based mold release system (WaterWorks Aerospace Release). Then the mixture is cured for 4 hours at $80{ }^{\circ} \mathrm{C}$ for gelation and 4 hours at $120{ }^{\circ} \mathrm{C}$ for post-curing. The maximum glass transition temperature is not realized with this curing cylce. Finally, the test specimens were cut out of the cured plates via a milling process.

\subsection{Test methods}

The experimental test results are statistically secured with 7 specimens for the tensile tests and 10 specimens for the fracture tests. The specimens were conditioned for at least 2 days at a temperature of $23{ }^{\circ} \mathrm{C}$ and a humidity of $51 \%$. A Zwick/Roell (Z005) static testing machine is used for mechanical testing. Concerning the tensile tests, specimens with a thickness of $2 \mathrm{~mm}$ are produced and tested according to testing standard DIN EN ISO 527-1 and DIN EN ISO 527-2, sample type 1B. The stress/strain curves are recorded with a test speed of $1 \mathrm{~mm} / \mathrm{min}$ and an initial load of $5 \mathrm{~N}$. The tensile modulus is evaluated at strain levels of $0.05 \%$ and $0.25 \%$, while defining a limit of $5 \%$ standard deviation for considering the results to be valid. Fracture toughness specimens are produced and tested according to ISO 13586 . The test speed is set to $10 \mathrm{~mm} / \mathrm{min}$ and the crosshead displacement and emerging forces during the testing are recorded. For evaluating the fracture toughness, the critical energy release rate $G$ of the pre-cracked specimens (precrack length between 7 and $13 \mathrm{~mm}$ ) is calculated. For compact tension (CT) specimen, the critical energy release rate is defined as the amount of energy that is released when the crack starts to grow. In terms of ISO 13586, the standard deviation must not exceed $12 \%$.

\subsection{Numerical methods and models}

The simulations presented in the following are carried out using the Molecular Dynamic Finite Element Method (MDFEM) (for further information see Nasdala et al. [20]). The MDFEM provides a framework for the calculation of classic Molecular Dynamics (MD) problems within the finite element method (FEM) (for a general overview on molecular dynamics in the context of the finite element method see Wackerfuß [21]). This approach is generally motivated by the simplification of multi-scale techniques as, in particular, the coupling of atomistic and continuum mechanics simulations can be efficiently performed within one software package. Furthermore, the efficient solvers as well as the pre- and post-processing tools of the FEM software can be used or integrated and thus, the effort in special in-house developed software is reduced. However, for the present study, we focus on pure MDFEM models that do not contain continuum regions. Following investigations are performed starting on pure epoxy unit cells and subsequently moving on to particlematrix unit cells generated with in-house software using Open Babel [22] and Packmol [23]. A schematic representation of the models is given in Fig. 2 .

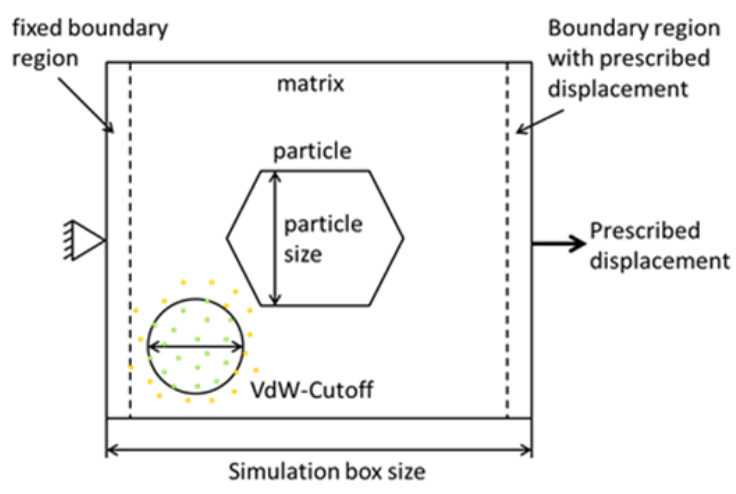

Fig. 2 Schematic representation of particle-matrix unit cells

The first step in the generation process of the chemical structure is the generation of the nanoparticles. For this purpose, the 
crystalline unit cell is duplicated and trimmed to the orthorhombic shape. MDFEM simulations, as well as MD simulations in general, require high numerical effort, and thus do not allow simulating the real primary particle size and even less the real agglomerates in intensive parametric studies. However, as shown in Fig. 3, preliminary studies indicate that particles with absolute sizes above $3 \mathrm{~nm}$ nearly not influence the elastic properties. Therefore, this particle size is used for all further simulations. As well as the rather minor error due to particle size, an additional error is due to not modeling agglomerates. Thus, the following results can be regarded as a principle study, not exactly reflecting the real material behavior, but pointing out the essential mechanisms. A more detailed discussion on the influence of particle size and agglomeration effects on mechanical properties will be addressed in future work.

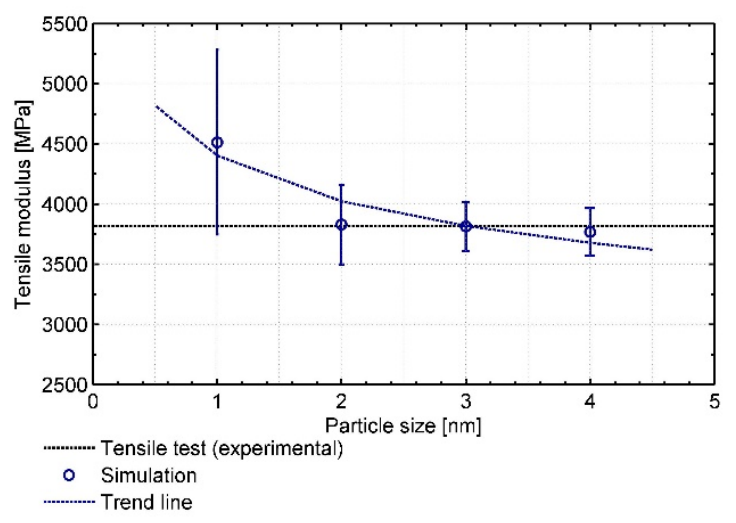

Fig. 3 Simulated influence of the primary particle size on the tensile modulus of the nanocomposite

Subsequently, a number of epoxy prepolymer chains, according to the specified weight proportions, are randomly packed into the simulation box and cross-linked using an algorithm similar to the work of Yarovsky and Evans [24] and Wu and $\mathrm{Xu}[25,26]$. In the present study, primarily the modeling of the particle surface modifications and the generation of the particle-matrix bonding is examined. The surface hydroxyl groups of unmodified boehmite particles are enabled to develop cross-links with the epoxy groups of the polymer during the cross-linking algorithm, and thus form chemical bonds between particle and matrix if the crosslinking conditions apply. Since no experimental data on the surface reactivity is available, particle surface hydroxyl groups are treated equal to polymer hydroxyl groups, i.e. the probability of forming a cross-link is equal. The acetic acid molecules are bonded to hydroxyl groups on the surface of the boehmite. Since the methyl group of the acetic acid molecule is non-reactive, it inhibits chemical bonding between particle and matrix in dependence on the loading ratio of the acetic acid, which is systematically varied in the following simulations. In the limit case of a complete surface loading, no chemical bonds between particle and matrix exist. For further information on the model generation, especially on the particle-matrix bonding of the unmodified boehmite, refer to the authors' previous work $[11,16]$.

The following simulations are, if not marked otherwise, performed using the MDFEM in combination with the DREIDING force field [27]. Different cutoff radii (denoted as VdWCutoff) have been applied for the particle, the matrix and between particle and matrix. According to [11] and [16], values of $8 \AA$ for the particle and $15 \AA$ for the matrix and between particle and matrix are used. After the model generation, a relaxation step is performed over a time period of 100 ps, guaranteeing a sufficient convergence of the virial stresses. Subsequently, a displacement of one of the boundary regions is prescribed. The external load rate is selected with particular regard to avoid undesirable dynamic effects. Maxima of $5 \%$ strain in a period of 500 ps for the pure epoxy and 3\% strain in a period of $400 \mathrm{ps}$ for the particle unit cells are selected from previous 
parametric studies. The chosen load rates result in a roughly equal acceleration. For relaxation as well as loading, all atoms within a range of $3 \AA$ from both boundaries in loading direction (see Fig. 2) are constrained to the simulation box in all directions. The atoms on all other boundaries are unconstrained (i.e. vacuum boundary conditions). Additionally, the box is constrained to remain in a rectangular shape.

\subsection{Preliminary simulations for pure epoxy}

The general aim of the numerical simulations is to provide methods for the prediction of material properties. In order to simulate nanocomposite materials, it is necessary to simulate the separated components, like the pure matrix material. Thus, in preliminary simulations, numerical tensile tests on pure epoxy resin were performed in order to calibrate simulation and modeling parameters, like the maximum cross-link distance of the epoxy curing algorithm. These simulations are the foundation for the realistic simulation of particle reinforced materials.

Fig. 4 exemplarily shows results of a study on the simulation box size of pure epoxy, where all parameters have already been adjusted. A curve calculated with the UFF force field [28] is shown for comparison. Considering the standard deviations (displayed by error bars in Fig. 4), for each curve results of three virtual tensile tests have been averaged. With increasing simulation box size, the simulation curve approaches the experimental curve, while the standard deviation significantly decreases. Satisfying agreement between simulation and experiment with a reasonably small deviation is reached at a box size of $10 \mathrm{~nm}$. In the nonlinear region above approximately $2.5 \%$ strain, small deviations are observable. This is caused by the harmonic potentials of the DREDING force field that have been used for efficiency and stability reasons. Thus, the simulations do not include the failure of bonds and tend to poorly reproduce the inelastic behavior compared to non-harmonic potentials. Furthermore, fracture of real polymer materials is closely related to stress concentrations due to micro-scaled material imperfections, like voids [29]. However, these imperfections are too big for the simulation using molecular dynamics. Thus, the numerical experiments are focused on the elastic behavior.

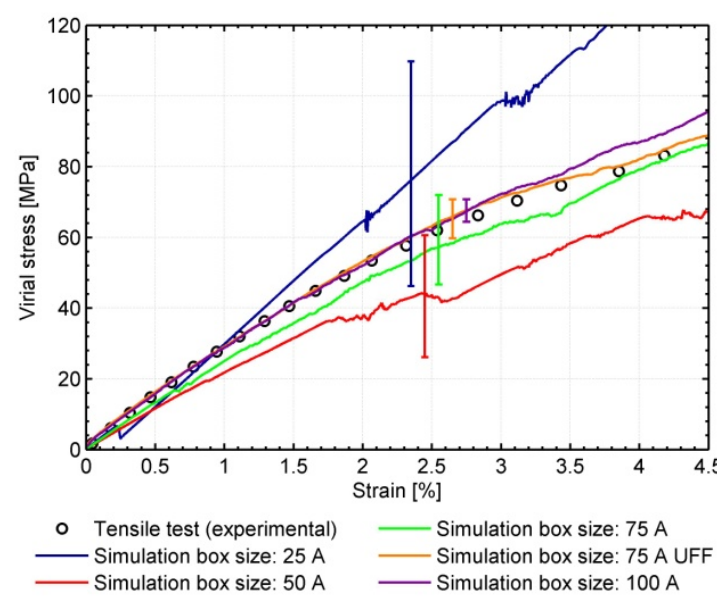

Fig. 4 Influence of the simulation box size on the mechanical response of the pure epoxy

In addition to the excellent accordance of the tensile behavior, the average final density of $1.16 \mathrm{~g} / \mathrm{cm}^{3}$ after relaxation shows a good agreement with the real material (approx. $1.19 \mathrm{~g} / \mathrm{cm}^{3}$, [30]). In the simulation model $94.06 \%$ of the epoxy groups are cross-linked. That is close to the experimental estimation of $97.5 \%$ via differential scanning calorimetry (DSC). However, a certain deviation between simulation and experiment is expected, since the DSC measures intensities of certain spectra (no absolute number of crosslinks), whereas numerical methods count exactly the number of cross-links. 


\section{Results and discussion}

\subsection{Tensile tests results}

The tensile properties were determined using test specimen with particle mass fractions between 0 and 15 wt\% for both particle configurations. Average values and standard deviations of the experimental test results are summarized in Table 1.

Table 1 Tensile modulus and ultimate stress for epoxy/boehmite nanocomposites at varying mass fractions and surface modifications (unmodified and acetic acid).

\begin{tabular}{|c|c|c|c|c|}
\hline \multirow[b]{2}{*}{ wt\% } & \multicolumn{2}{|c|}{$\begin{array}{l}\text { Unmodified } \\
\text { boehmite }\end{array}$} & \multicolumn{2}{|c|}{$\begin{array}{l}\text { Acetic acid } \\
\text { boehmite }\end{array}$} \\
\hline & $\begin{array}{l}\mathrm{E}_{\mathrm{TEN}} \\
{[\mathrm{MPa}]}\end{array}$ & $\begin{array}{l}\sigma_{\text {ultimate }} \\
{[\mathrm{MPa}]}\end{array}$ & $\begin{array}{l}E_{\text {TEN }} \\
{[\mathrm{MPa}]}\end{array}$ & $\begin{array}{l}\sigma_{\text {ultimate }} \\
{[\mathrm{MPa}]}\end{array}$ \\
\hline 0.0 & $\begin{array}{l}3333 \pm \\
17\end{array}$ & $\begin{array}{l}87.31 \\
\pm 1.28 \\
\end{array}$ & $\begin{array}{l}3333 \pm \\
17\end{array}$ & $\begin{array}{l}87.31 \quad \pm \\
1.28\end{array}$ \\
\hline 1.0 & & & $\begin{array}{l}3427 \pm \\
30\end{array}$ & $\begin{array}{l}85.02 \quad \pm \\
0.97\end{array}$ \\
\hline 2.5 & $\begin{array}{l}3402 \pm \\
31\end{array}$ & $\begin{array}{l}80.31 \\
\pm 6.20\end{array}$ & $\begin{array}{l}3491 \pm \\
22\end{array}$ & $\begin{array}{ll}82.73 \quad \pm \\
3.52 & \end{array}$ \\
\hline 5.0 & $\begin{array}{l}3487 \pm \\
24\end{array}$ & $\begin{array}{l}81.72 \\
\pm 7.20 \\
\end{array}$ & $\begin{array}{l}3633 \pm \\
14\end{array}$ & $\begin{array}{l}83.77 \quad \pm \\
3.40\end{array}$ \\
\hline 10.0 & $\begin{array}{l}3751 \pm \\
33\end{array}$ & $\begin{array}{l}83.10 \\
\pm 2,80\end{array}$ & $\begin{array}{l}3915 \pm \\
16\end{array}$ & $\begin{array}{l}82.14 \quad \pm \\
6.28\end{array}$ \\
\hline 15.0 & $\begin{array}{l}4009+ \\
31\end{array}$ & $\begin{array}{l}84.20 \\
\pm 1.60 \\
\end{array}$ & $\begin{array}{l}4185 \pm \\
22\end{array}$ & $\begin{array}{ll}81.89 & \pm \\
8.14 & \end{array}$ \\
\hline
\end{tabular}

Fig. 5 shows the tensile modulus depending on the particle mass fraction. With increasing mass fraction the tensile modulus increases nearly linearly for both particle configurations, substantiated by a correlation coefficient of 0.993 for unmodified boehmite composites and 0.999 for acetic acid modified boehmite composites. The tensile modulus amounts $3333 \mathrm{MPa}$ for neat epoxy, $4009 \mathrm{MPa}$ for 15 wt\% unmodified boehmite composites and $4185 \mathrm{MPa}$ for $15 \mathrm{wt} \%$ acetic acid modified boehmite composites. Referring to neat epoxy, this is a percentage increase of $20 \%$ and $26 \%$, respectively. The standard deviations are below $1 \%$. Composites with acetic acid modified boehmite have an increased tensile modulus compared to composites with unmodified boehmite, while the effect of surface modification increases with rising particle content up to $6 \%$ (200 $\mathrm{MPa})$.

In general the increased Young's modulus results from the stiffer boehmite particles. However, particle-matrix interactions must be considered, to explain the effect of surface modification. Since the acetic acid is nonreactive, the epoxy is assumed to crosslink undisturbed until reaching the particle surface, leading to a stiffer or rather no interphase (increased cross link density). Thus, with increasing particle mass fraction the volume fraction of the stiffer interphase increases, too, causing the almost linearly increasing deviation in overall Young's modulus between composites with unmodified and acetic acid modified boehmite. The effect of acetic acid might be amplified further by increasing the surface loading. Furthermore there might be nonbonded acetic acid molecules that become accessible due to agglomerate fracture during dispersion. These free molecules could react with molecules of the resin system, negatively affecting the epoxy cross-linking. This might induce a lowered increase in overall Young's modulus and weaken the effect on the increased interphase stiffness.

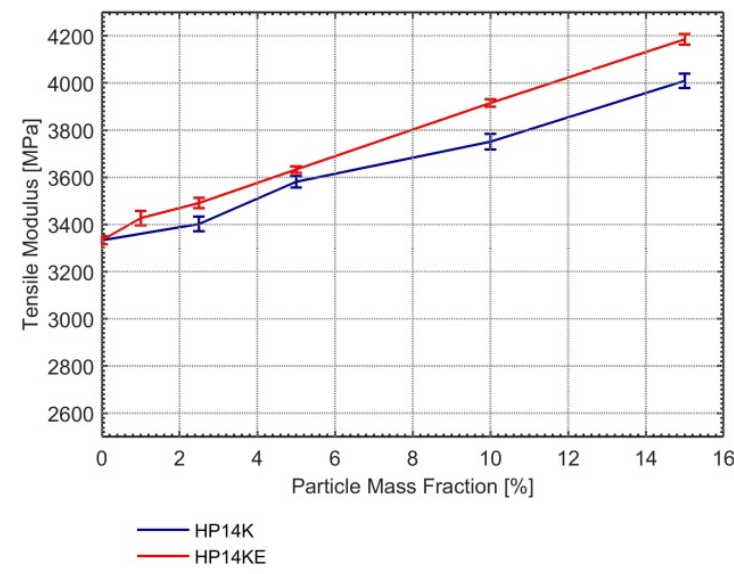

Fig. 5 Tensile modulus for epoxy/boehmite nanocomposites as function of mass fraction and surface modifications. Conditions: constant average particle size of $105 \mathrm{~nm}$; surface modification: a) unmodified boehmite (HP14K) and b) acetic acid modified boehmite (HP14KE). 
Fig. 6 shows the tensile strength related to the particle mass fraction for unmodified and acetic acid modified boehmite. For both particle configurations the tensile strength is not significantly affected by the particle content and decreases only marginally. In comparison to the unfilled epoxy resin the tensile strength for acetic acid modified boehmite composites decreases by maximum $6 \%$ and for unmodified boehmite composites by maximum $8 \%$. In general, the tensile strength shows comparable values for both particle configurations. The average values slightly decrease for acetic acid modified boehmite composites at weight fractions above 5 wt\%, whereas for unmodified boehmite composites the tensile strength slightly increases at weight fractions above 2.5 wt\%.

Decreasing values of tensile strength for acetic acid modified boehmite composites might be indeed indicating that acetic acid leads to weaker particle-matrix bonding and thus, decreased interfacial stress transfer. However, changes in tensile strength are within the range of standard deviations for both particle configurations, reducing the significance of the test result trends. In summary, the tensile strength of the investigated composites is not significantly affected by particle content and surface modification. Though, the results are in accordance with the dynamic light scattering measurements, indicating that particle distributions and agglomerate sizes for both particle configurations are roughly the same. Results presented in previous work [17], showing that especially the tensile strength depends on agglomerate size, substantiate this statement.

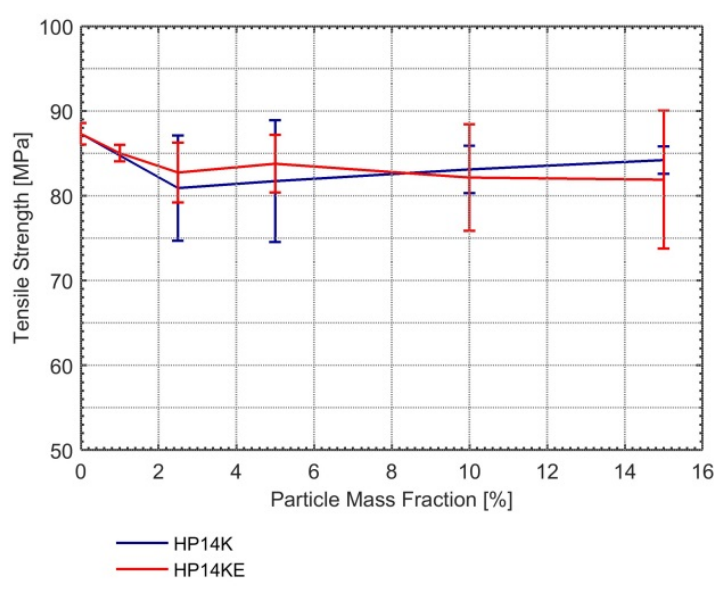

Fig. 6 Tensile strength for epoxy/boehmite nanocomposites as function of mass fraction and surface modifications. Conditions: constant average particle size of $105 \mathrm{~nm}$; surface modification: a) unmodified boehmite (HP14K) and b) acetic acid modified boehmite (HP14KE).

\subsection{Fracture toughness test results}

The fracture behavior of boehmite/epoxy composites was investigated regarding the nanoparticle content and the surface modification with the help of compact tension (CT) specimens. The measured critical energy release rates $\left(G_{\mid c}\right)$ are listed in Table 2. For both particle configurations, $G_{\mid c}$ increases with rising nanoparticle content. The results are in good accordance with studies of Wetzel et al. [31] and Adachi et al. [32].

Table 2 Critical energy release rate for epoxy/boehmite nanocomposites at varying mass fractions and surface modifications (unmodified and acetic acid).

\begin{tabular}{|l|l|l|}
\hline & Unmodified & Acetic acid \\
\hline$w t \%$ & $G_{\mathrm{Ic}}\left[\mathrm{kJ} / \mathrm{m}^{2}\right]$ & $\mathrm{G}_{\mathrm{Ic}}\left[\mathrm{kJ} / \mathrm{m}^{2}\right]$ \\
\hline 0.0 & $0.109 \pm 0.007$ & $0.109 \pm 0.007$ \\
\hline 1.0 & $0.136 \pm 0.013$ & $0.134 \pm 0.008$ \\
\hline 2.5 & $0.138 \pm 0.016$ & $0.140 \pm 0.016$ \\
\hline 5.0 & $0.155 \pm 0.011$ & $0.151 \pm 0.012$ \\
\hline 10.0 & $0.164 \pm 0.010$ & $0.173 \pm 0.025$ \\
\hline 15.0 & $0.176+0.008$ & $0.177+0.009$ \\
\hline
\end{tabular}

In general, the influence of particle reinforcement on fracture toughness is considerably higher than on tensile properties. For a particle content of $15 \mathrm{wt} \%$, 
the increase in $\mathrm{G}_{\mathrm{lc}}$ referred to the neat epoxy amounts approximately $62 \%$. In contrast to the tensile modulus, the influence on fracture toughness due to particle reinforcement is nearly 3 times higher. Fig. 7 shows the critical energy release rate $\left(G_{c c}\right)$ for both particle configurations depending on the particle content. Particularly noticeable is the sudden increase in $G_{\mid c}$ comparing neat epoxy to epoxy with $1 \mathrm{wt} \%$ particles. Above a mass fraction of $1 \mathrm{wt} \%, \mathrm{G}_{\mathrm{lc}}$ increases nearly linearly with rising particle content, substantiated by correlation coefficients of 0.976 for reinforcement with unmodified boehmite and 0.970 for reinforcement with acetic acid modified boehmite. For both particle configurations the test results are in the same range and the standard deviations overlap.

The results indicate that acetic acid substantially has no influence on the fracture behavior. The effect of the overall changed crosslink density due to surface modification (as previously discussed in connection with the tensile modulus) on the fracture toughness might be dominated by toughening effects, like crack deflection, particle debonding and crack pinning. However, also the fracture toughness test results correlate with the dynamic light scattering measurements, showing almost identical values for both particle configurations, indicating that the agglomeration rate is the same for both particle configurations. In addition to mechanical tests, SEM-micrographs of fracture surfaces are evaluated to characterize the influence of acetic acid modification and the dominating toughening mechanisms.

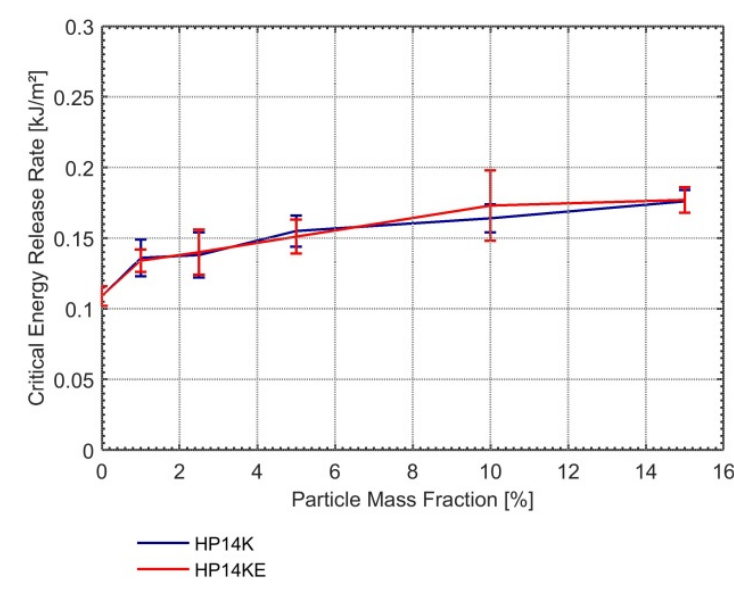

Fig. 7 Critical energy release rate for epoxy/boehmite nanocomposites as function of mass fraction and surface modifications. Conditions: constant average particle size of $105 \mathrm{~nm}$; surface modification: a) unmodified boehmite (HP14K) and b) acetic acid modified boehmite (HP14KE).

4.3 Fracture surfaces - toughening mechanisms

Size and strength of the particle as well as the particle-matrix interaction determine the occurring toughening mechanism. A high particle stiffness and strong particle-matrix interactions should lead to crack deflection and crack pinning, whereas weak particlematrix interactions should lead to de-bonding effects. Due to size and stiffness of the boehmite particles, a particle bridging effect can be excluded. Particle bridging can be found in composites with elastic or large particles, where the particles act as stress transfering link between the flanks of the crack. A significant influence on the fracture toughness is attributed to crack pinning. Especially highly stiff particles are able to pinn the crack front, since they force the crack to bypass the particle. Therefore the distance between the particles and thus, the particle volume fraction has major influence on the crack pinning mechanism. However, due to the huge difference between nanoparticle and crack tip size, it is doubtable if the boehmite nanoparticles are able to pin the crack front. In fact, in the framework of 
this study, the characteristic curved lines indicating crack pinning could not be found.

If highly stiff particles are strongly bond to the matrix, the crack can be deflected by the particle and proceed in another direction. Due to crack growth the repeatively deflection of the crack leads to an increased fracture surface and energy absorption. Generally, the roughness of fracture surfaces increases with increasing particle content. The possibility a crack front passing a particle increases with rising mass fraction, simply because the number of particles increases. As a result, the chance for occurring crack deflections increases. If the strength of the particle-matrix bonding is higher than the particle fracture strength the crack breaks the particle. However, in nanocomposites with agglomerated particles it is likely to appear but questionable, if fractured agglomerates look different than smaller agglomerates that managed to withstand a fracture. Particles de-bond due to low particle-matrix interactions. The process of particle debonding only absorbs small amounts of energy. Nevertheless, the degree of debonding can be an indicator for the strength of partice-matrix interaction. Holes in the fracture surface are indicators for particle debonding, since de-bonded particles produce voids. The missing particle could either be found on the opposite fracture surface or has been dropped out of the matrix during fracture.

Fig. 8 and Fig. 9show fracture surfaces of CTspecimen with a) 5 wt\% unmodified boehmite and b) 5 wt\% acetic acid modified boehmite. The micrographs show areas of the fracture surfaces that are approximately 15 $\mathrm{mm}$ behind the initial crack. Fig. 8 shows lower resolved SEM micrographs compared to Fig. 9. However, these micrographs give a better overview of the fracture surface. Compared to the fracture surface of unmodified boehmite composites, the fracture surface of the acetic acid boehmite composite (b) shows clearly more voids on the fracture surface, presumably caused by a de-bonding of particle and matrix (exemplarily marked by arrows in Fig. 8). Higher resolved SEM micrographs (Fig. 9) confirm that voids primarily occur in the direct proximity of a particle (exemplarily marked by circles in Fig. 8). The frature surfaces indicate that the acetic acid modification increases the de-bonding probability, due to a weakening of the particle-matrix interaction. 


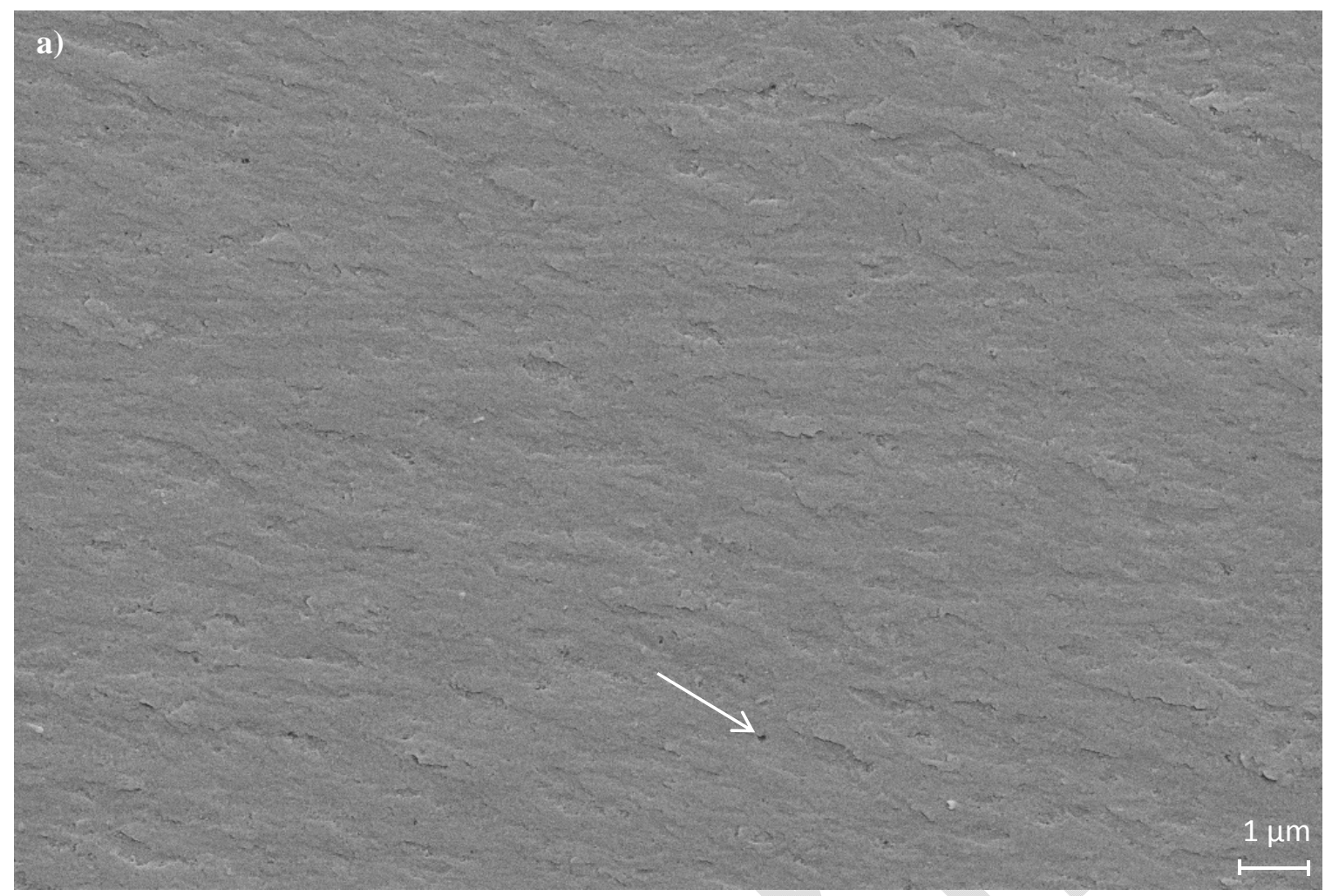

b)

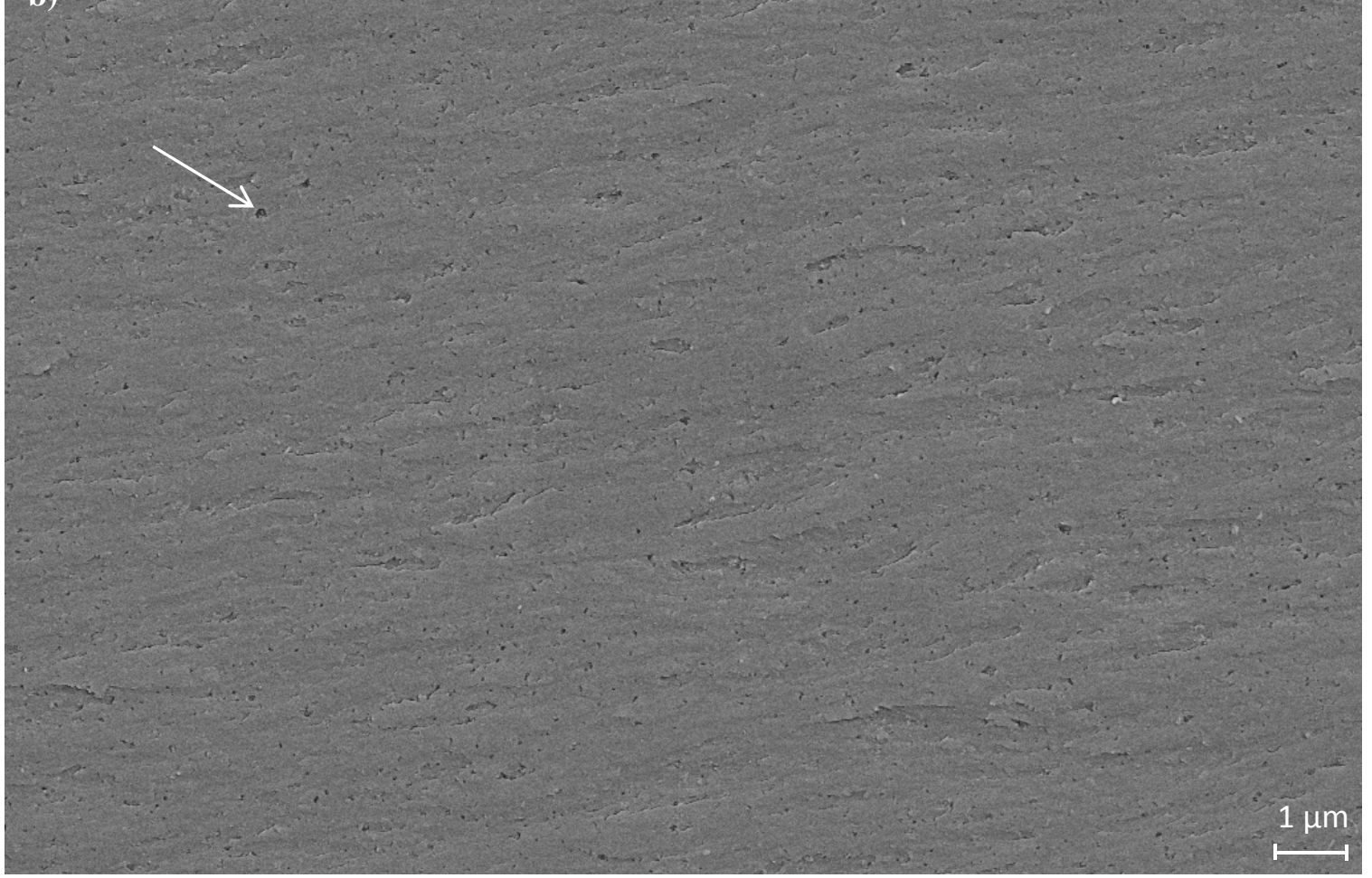

Fig. 8 SEM micrographs of fracture surfaces (CT-specimen) for composites with a) $5 \mathrm{wt} \%$ unmodified, b) $5 \mathrm{wt} \%$ acetic acid modified boehmite 

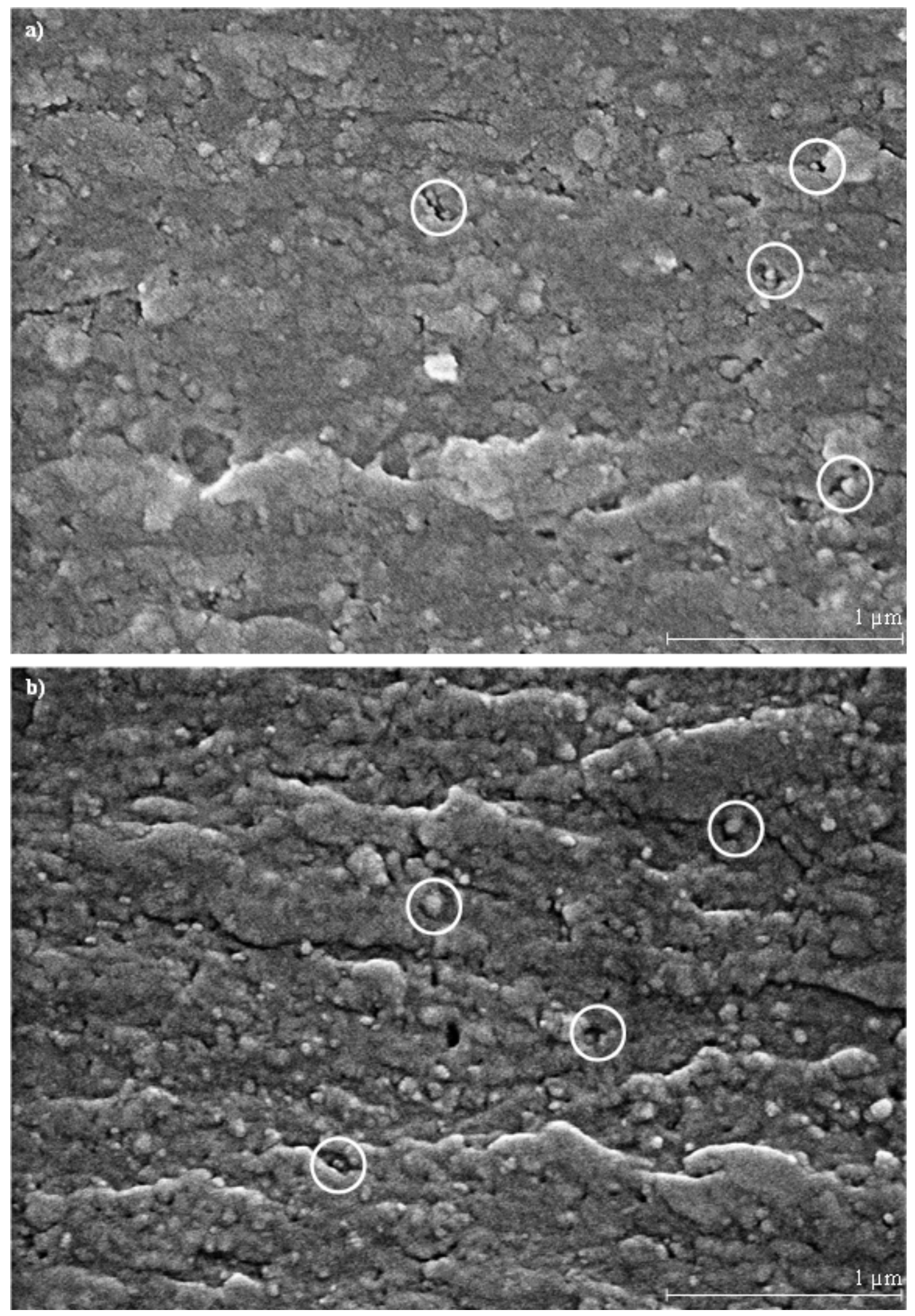

Fig. 9 SEM micrographs of fracture surfaces (CT-specimen) for composites with a) 5 wt\% unmodified, b) 5 wt $\%$ acetic acid modified boehmite 


\subsection{Nanocomposite simulations}

Besides providing methods for the prediction of material properties in the sense of a virtual material design, aims of the simulations are the determination of main mechanisms by which acidic acid affects mechanical properties and to indicate possible causes of partly unexpected experimental behavior. As stated before, the present study only focuses on elastic behavior.

Fig. 10 shows the results of a parametric study on the effect of the particle weight fraction and surface modification on the nanocomposite's Young's modulus. Each bar represents the average of 9 calculations ( 3 per spatial direction). The simulation was performed assuming that the particle surface is completely loaded with acetic acid molecules and consequently no particlematrix bonds are present. The experimentally measured increase of Young's modulus due to rising mass fraction can be reproduced well for unmodified boehmite. In contrast, the acetic acid modified boehmite shows distinct deviations for all weight fractions. It features only a slight increase of the Young's modulus compared to the unfilled matrix and a substantial deviation between the simulations. In summary, the simulation results of the acetic acid modified boehmite show a rather poor agreement with the experiments.

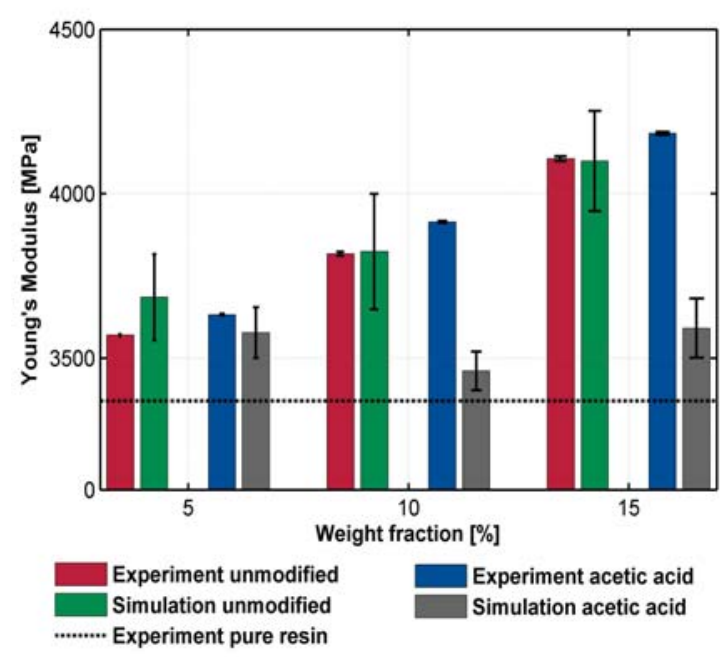

Fig. 10 Parametric study on the influence of the particle weight fraction and the surface modification on the resulting Young's Modulus of the nanocomposite

However, these simulations indicate that the increase of the elastic properties is mainly induced through a strong particle-matrixbonding. In particular, the positive effect due to a strong particle matrix bonding dominates the negative effect caused by a softening in the particle-matrix-interphase region due to the reduction of the epoxy cross-links. In the real material, additional effects not considered in the simulation are likely to occur (see also Section 5) causing the deviation between the simulation and the measurements.

To investigate the influence of surface modification loading, models with partial surface loading were generated by modifying the procedures outlined in section 3.3. In order to control the surface loading, a spacing radius is introduced. If an acetic acid molecule is added to the model, no other molecule can be placed within its spacing radius. In contrast to a percentage of surface loading, this approach adds physical meaning in the sense of repulsive forces due to partial charges of the molecules. An overview of the resulting composition of the particle surface for different spacing radii is given as an average of all models in Table 3. Therefore, the particle surface hydroxyl groups are divided into three groups: those that are 
covered by molecules of the surface modification, those that are not covered and form particle-matrix bonds and those that are not covered and do not form particle-matrix bonds (denoted as non-bonded surface hydroxyl groups). As expected, the number of non-bonded surface hydroxyl groups increases and the number of surface hydroxyl groups covered by acetic acid molecules decreases quadratically with the spacing radius. However, the amount of particlematrix bonds increases non-proportionally to the increase of surface hydroxyl groups.

Table 3 Composition of the particle surface (204 total surface hydroxyl groups) regarding the spacing radius (spacing radius: if an acetic acid molecule is placed, no other molecule can be placed within the spacing radius)

\begin{tabular}{|c|c|c|c|}
\hline $\begin{array}{c}\text { Spacing } \\
{[\AA]}\end{array}$ & $\begin{array}{c}\text { Particle-matrix } \\
\text { bonds [\%] }\end{array}$ & $\begin{array}{c}\text { Non-bonded surface } \\
\text { hydroxyl groups [\%] }\end{array}$ & $\begin{array}{c}\text { Surface hydroxyl groups covered } \\
\text { by acetic acid molecules [\%] }\end{array}$ \\
\hline 2.5 & 0 & 3.9 & 96.1 \\
\hline 5 & 1.3 & 48.7 & 50.0 \\
\hline 7.5 & 3.3 & 73.7 & 23.0 \\
\hline 10 & 6.9 & 82.3 & 10.8 \\
\hline Unmodified boehmite & 14.4 & 85.6 & 0 \\
\hline
\end{tabular}

Fig. 11 shows the simulation results of the Young's modulus depending on surface loading for a constant mass fraction of 10 wt\%. To account for the small differences in the resulting Young's moduli, the number of averaged simulation boxes has been increased to 18 (six per spatial direction) in order to reduce the statistical spread. The Young's modulus increases approximately linearly with the spacing radius, starting at values only slightly above the stiffness of neat epoxy for a spacing radius of $2.5 \AA$ up to the stiffness of unmodified boehmite for an imaginary infinite spacing radius. Since all degrees of surface loading, as expected, lead to a reduction in elastic properties, the reduced surface loadings cannot explain the experimental result for the acetic acid modified boehmite.

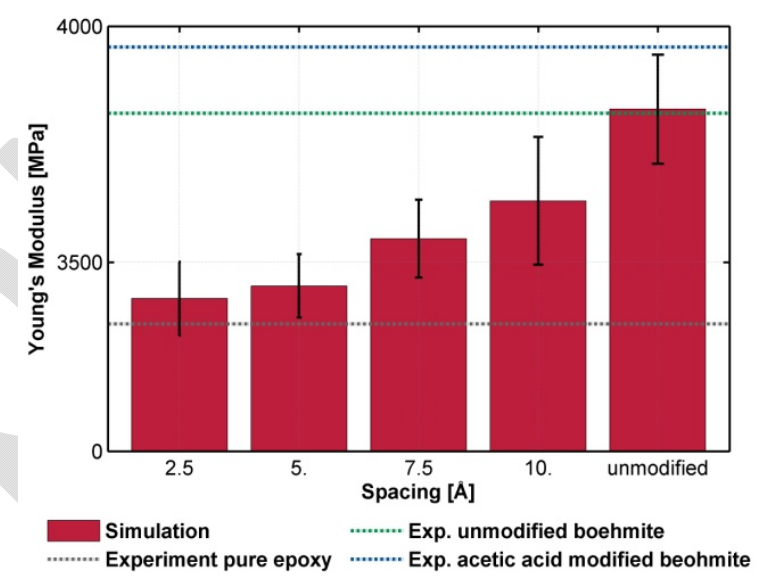

Fig. 11 Parametric study on the influence of the spacing between acetic acid molecules on the resulting Young's Modulus of the nanocomposite containing 10 wt\% boehmite particles

Even though the simulations of the acetic acid modified boehmite still predict Young's moduli different from the experiment, in the following the influence of the acetic acid modification on local properties of the particle-matrix interphase is investigated for the case of complete surface loading. Thus, the following results can be regarded as a principle study on the underlying mechanisms due to surface modification that lowers the chemical bonding between particle and matrix. The following results are the outcome of the model generator and especially the cross-linking algorithm (addressed in section 3.3). The algorithm, 
originally applicable to pure resin, was expanded to include particle-matrix crosslinking and is able to account for competition between particle-matrix cross-linking and epoxy to epoxy cross-linking in the surrounding matrix. Fig. 12 shows the results for the radial density (a), the cross-link density (i.e. cross-links per volume) between epoxy pre-polymer chains (b), the total crosslink density (including particle-matrix crosslinks (c) and hardener molecules per volume (d).

The unmodified boehmite shows a significant accumulation of hardener molecules in the vicinity of the particle surface (approx. 17-21 $\AA$ from the particle center of mass) and a slight depletion in the region between approximately 20 and $25 \AA$ (d). Fig. 12 a) also shows a slight reduction in radial density of the unmodified boehmite in the region of 20$25 \AA$, indicating an expulsion of epoxy chains in this region. This is in good agreement with (b) and (c). Fig. 12 c) shows that the total cross-link density quickly reaches the value of the bulk epoxy, whereas the comparison to b) indicates that below $25 \AA$, a significant number of cross-links are created between particle and matrix instead of between two epoxy pre-polymer chains.

The acetic acid modified boehmite exhibits a huge density reduction in the vicinity of the particle surface $(18-23 \AA)$ caused by the presence of acetic acid molecules and the expulsion of epoxy in this region. Apart from that, the acetic acid produces an undisturbed epoxy network, as there are no particlematrix cross-links, and thus the total crosslink density and the epoxy cross-link density are equal (b) and c). The total cross-link density in comparison to the unmodified boehmite shows a shift of approximately the same size as the low region in the radial density plot, which is caused by the expulsion of the epoxy by the acetic acid molecules (c). Fig. $12 \mathrm{~d}$ ) additionally shows that there is no accumulation or depletion of hardener molecules, but the value of the bulk epoxy is reached immediately. 

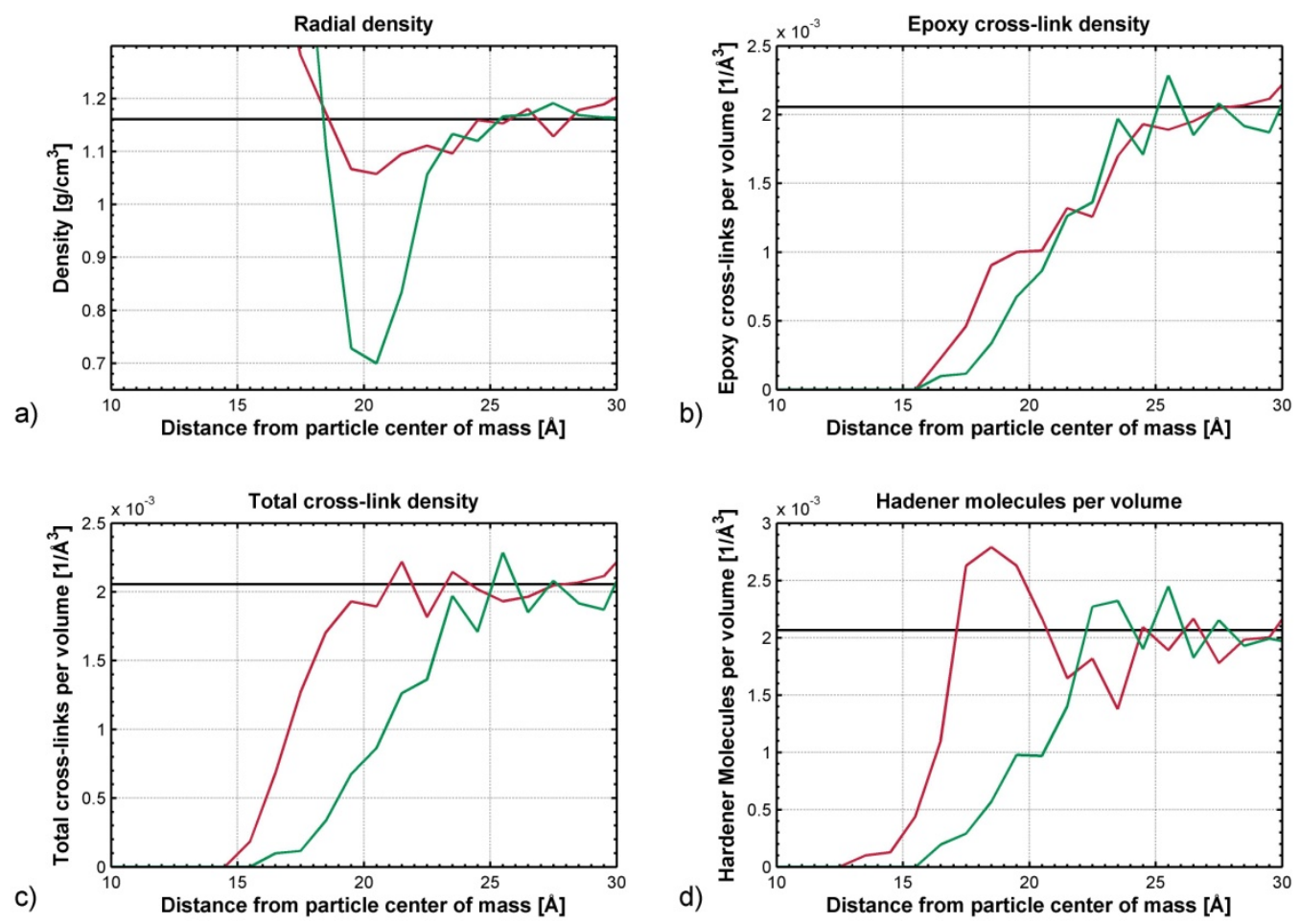

- Bulk epoxy Unmodified boehmite

Acetic acid modified boehmite

Fig. 12 Comparison of the local interphase morphology of the relaxed epoxy with $10 \mathrm{wt} \%$ unmodified boehmite (red) and with 10 wt\% acetic acid modified boehmite (green).

In total, four phenomena can be observed and are displayed schematically as a series connection of springs in Fig. 13. Firstly and secondly, the acetic acid degrades the particle-matrix bonding and leads to a less disturbed epoxy network in the vicinity of the particle. This leads to an effect on the stiffness, expressed by the drastic decrease of the stiffness of the P-M spring and the absence of the INT spring in the case of the acetic acid system. Thirdly, there is an additional effect of the expulsion of the epoxy due to the presence of the acetic acid molecules. This leads to a region of low density around the particle that should also lead to a decrease in stiffness. Thus, for the acetic acid, there is the additional SM spring, that possesses a lower level of stiffness compared to the bulk epoxy. Finally, the surface modification adds mass to the particles and is responsible for a slight decrease in the actual particle mass fraction that should also result in reduced stiffness of the homogenized material. It should be mentioned again that Fig. 13 only represents a qualitative statement on the composition of the nanocomposite material. Quantitative information on the stiffness of each component cannot be obtained from the comparison of the effective stiffness of the nanocomposite (i.e. the complete series connection) to the measurements. In particular, the presence of agglomerates leads to a change in the stiffness of the particle placeholder in Fig. 13 and strongly influences the overall behavior. 


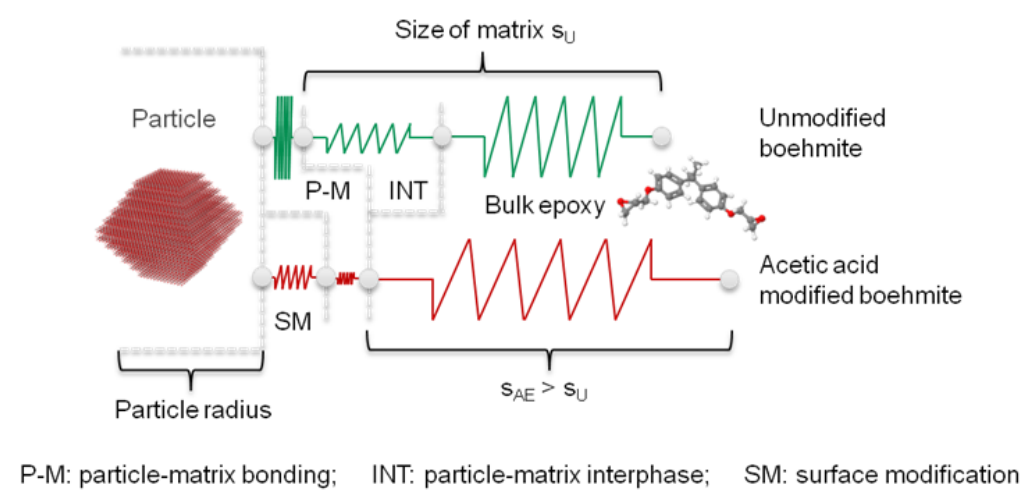

Fig. 13: Schematic representation of the influence of acetic acid on the stiffness of the nanocomposite (vertical extent of the springs depicts the size of the stiffness)

\section{Concluding remarks}

The experimental test results show a substantial increase in the mechanical properties of epoxy/boehmite nanocomposites in comparison to the unfilled epoxy system, as the tensile modulus increases between 20 and $26 \%$ and the fracture toughness by approximately $62 \%$. While there is a good correlation between the experimental and numerical results for unmodified boehmite, the results for acetic acid modified boehmite do not match. In order to find explanations for the difference, numerical investigations of the interphase morphology were performed. In the following, the results are discussed with respect to the hypotheses formulated in the introduction (section 1).

In Hypothesis 1 it was stated that the acetic acid weakens the particle-matrix bonding and reduces the size of the particle-matrix interphase by enabling the epoxy to cross-link unhindered until reaching the phase boundary. This hypothesis is substantiated by the evaluation of the curing statistics of the numerical models, showing considerable changes in the epoxy morphology in the vicinity of the unmodified boehmite nanoparticles. Furthermore, this assumption is in accordance with the tensile test results, assuming that the increased degree of crosslinking in the vicinity of the particles might leads to an overall increase in stiffness. The results show that the acetic acid leads to an increase in tensile modulus of nearly $6 \%$ for a particle mass fraction of $15 \mathrm{wt} \%$.

Hypothesis 2 states that the weakened particle-matrix bonding dominates the change in the degree of cross-linking and thus the overall stiffness of the acetic acid modified boehmite composite is lower compared to the stiffness of the unmodified boehmite composite. This is in accordance with the simulation but could not be corroborated by the mechanical tests. This indicates that the hypothesis and thus the simulation do not cover all aspects of the real behavior of the acetic acid modified boehmite composite.

Hypothesis 3 regards the effect of the acetic acid modification on the tensile strength. It is hypothesized that the weaker particle-matrix bonding lowers the interfacial stress transfer and thus the maximum tensile strength. The hypothesis could not be confirmed by mechanical tests. Generally, it has been found that the tensile strength is not affected significantly by the acetic acid modification. Measured differences in tensile strength are within the standard deviation, indicating that the particle size distribution for both particle systems is the same.

Hypothesis 4 concerns the influence of the acetic acid on the fracture behavior and 
states that the weakened particle-matrix bonding leads to a higher chance of particle de-bonding. Even though the critical energy release rate is the same for both particle systems, the fracture surfaces show obvious differences. There are clearly more voids on the fracture surfaces of the composites with acetic acid modified boehmite, indicating that hypothesis 4 is valid.

In summary, the difference between the unmodified and the acetic acid modified system is lower than expected for all tests. While hypothesis 1 and 4 could be confirmed or at least substantiated, hypothesis 2 and 3 seem to be invalid. The discrepancies between the hypotheses and the results obtained in the framework of this study will be addressed in future work. Possible, unproven aspects are listed in the following.

1. The inner surfaces of the agglomerates might be less modified. During dispersion, this would lead to an increased surface area without acetic acid modification.

2. The acetic acid desorbs from the boehmite surface, resulting in unmodified boehmite particles. The desorbed molecules could furthermore induce additional effects on the cross-linking of the polymer.

3. The domination of the particlematrix-bonding effect over the particle-matrix-interphase effect shown in the simulations could be reversed for significantly softer agglomerates. To be precise, for a soft reinforcement phase, the influence of the reduction of the interphase stiffness in case of the unmodified boehmite on the homogenized stiffness might be lower than the influence of the weakened chemical interaction between agglomerate and matrix in the presence of acetic acid.

\section{Acknowledgements}

This work originates from the Research Unit FOR 2021: "Acting Principles of Nano-Scaled Matrix Additives for Composite Structures", funded by the German Research Foundation (DFG). Furthermore, the authors acknowledge the support by the LUIS scientific computing cluster, which is funded by the Leibniz Universität Hannover, the Lower Saxony Ministry of Science and Culture (MWK) and the DFG. The authors wish to express their gratitude for the financial support. The authors also gratefully acknowledge the support from the company Sasol Germany $\mathrm{GmbH}$ for providing the modified and non-modified boehmite nanoparticles, for the technical support from the Institute of Materials (IFW) of the Technical University Braunschweig concerning the investigation of fracture surfaces via SEM and the Institute of Composite Structures and Adaptive Systems of the German Aerospace Center (DLR) Braunschweig concerning the preparation of the specimens and the implementation of the experimental tests.

\section{References}

[1] S. Mishra, S.H. Sonawane, R.P. Singh. Studies on characterization of nano CaCO3 prepared by the in situ deposition technique and its application in PP-nano CaCO3 composites. J POLYM SCI POLYM PHYS. 2005. 43:107-13.

[2] J. Douce, J.P. Boilot, J. Biteau, L. Scodellaro, A. Jimenez. Effect of filler size and surface condition of nano-sized silica particles in polysiloxane coatings. THIN SOLID FILMS. 2004.466:114-22. 
[3] S.Y. Fu, X.Q. Feng, B. Lauke, Y.W. Mai. Effects of particle size, particle/matrix interface adhesion and particle loading on mechanical properties of particulate-polymer composites. COMPOS PART B-ENG. (2008). 39: 933-961.

[4] R.P. Singh, M. Zhang, D. Chan. Toughening of a brittle thermosetting polymer: Effects of reinforcement particle size and volume fraction. J MATER SCI. (2002). 37:781-788.

[5] S. Kango, S. Kalia, A. Celli, J. Njuguna, Y. Habibi, R. Kumar. Surface modification of inorganic nanoparticles for development of organic-inorganic nanocomposites-A review. PROG POLYM SCl. (2013). 38: 1232- 1261.

[6] C. Arlt. Wirkungsweisen nanoskaliger Böhmite in einem Polymer und seinem Kohlenstofffaserverbund unter Druckbelastung. Dissertation. 2011.

[7] M.F. Uddin, C.T. Sun. Strength of unidirectional glass/epoxy composite with silica nanoparticleenhanced matrix. COMPOS SCI TECHNOL. (2008) 68(7-8), 1637-1643.

[8] A.K. Subramaniyan, C.T. Sun. Enhancing compressive strength of unidirectional polymeric composites using nanoclay. COMPOS PART A-APPL S. (2006). 37(12): 2257-2268.

[9] N. Shahid, R.G. Villate, A.R. Barron. Chemically functionalized alumina nanoparticle effect on carbon fiber/epoxy composites. COMPOS SCI TECHNOL. (2005). 65(14): 2250-2258.

[10] N.A. Siddiqui, R.S.C. Woo, J.K. Kim, C.C.K. Leung, A. Munir. Mode I interlaminar behavior and mechanical properties of CFRPs with nanoclay-filled epoxy matrix. COMPOS PART A-APPL S. (2007). 38(2): 449-460.

[11] J. Fankhänel, A. Kempe, R. Rolfes. Simulating Atomic Force Microscopy for the determination of the elastic properties of nano particle reinforced Epoxy resin. Proceedings ECCOMAS 2016. 2016.

[12] K.W. Putz, M.J. Palmeri, R.B. Cohn, R. Andrews, L.C. Brinson. Effect of Cross-Link Density on Interphase Creation in Polymer Nanocomposites. MACROMOLECULES. (2008) 41: 6752-6756.

[13] X. Bokhimi, J.A. Toledo-Antonio, M.L. Guzman-Castillo, F. Hernandez-Beltran. Relationship between crystallite size and bond lengths in boehmite. J SOLID STATE CHEM. (2001). 159: 3240.

[14] B. Finke, Institute for Particle Technology, TU Braunschweig (private communication).

[15] Jmol: open-source Java viewer for chemical structures in 3D. http://www.jmol.org/.

[16] J. Fankhänel, D. Silbernagl, M. Ghasem Zadeh Khorasani, B. Daum, A. Kempe, H. Sturm, R. Rolfes. Mechanical Properties of Boehmite Evaluated by Atomic Force Microscopy Experiments and Molecular Dynamic Finite Element Simulations. J NANOMATER. (2016). Volume 2016: 113.

[17] M. Jux, B. Finke, T. Mahrholz, M. Sinapius, A. Kwade, C. Schilde. Effects of Al(OH)O nanoparticle agglomerate size in epoxy resin on tension, bending and fracture properties. J NANOPART RES. (2017). 19: 139.

[18] M.J. Palimi, M. Rostami, M. Mahdavian, B. Ramezanzadeh. Surface modification of $\mathrm{Fe}_{2} \mathrm{O}_{3}$ nanoparticles with 3-aminopropyltrimethoxysilane (APTMS): An attempt to investigate surface treatment on surface chemistry and mechanical properties of polyurethane $/ \mathrm{Fe}_{2} \mathrm{O}_{3}$ nanocomposites. APPL SURF SCI. (2014). 320: 60-72.

[19] A.A. Javidparvar, B. Ramezanzadeh, E. Ghasemi. Effects of surface morphology and treatment of iron oxide nanoparticles on the mechanical properties of an epoxy coating. PROG ORG COAT. (2016). 90: 12-20.

[20] L. Nasdala, A. Kempe, R. Rolfes. The Molecular Dynamic Finite Element Method. CMC. (2010). 19(1). 57-104. 
[21] J. Wackerfuss. Molecular mechanics in the context of the finite element method. INT J NUMER METH ENG. (2009). 77(7): 969-997.

[22] N.M. O'Boyle, M. Banck, C.A. James, C. Morley, T. Vandermeersch, G.R. Hutchison. Open Babel: An open chemical toolbox. J CHEMINF. (2011). 3-33.

[23] L. Martínez, R. Andrade, E. G. Birgin, J. M. Martínez. Packmol: A package for building initial configurations for molecular dynamics simulations. J COMP CHEM. (2009) 30(13), 2157- 2164.

[24] I. Yarovsky, E. Evans. Computer simulation of structure and properties of crosslinked polymers: application to epoxy resins. Polymer. (2002). 43(3): 963-969.

[25] C. Wu, W. Xu. Atomistic molecular modelling of crosslinked epoxy resin. POLYMER. (2006). 47(16): 6004-6009.

[26] C. Wu, W. Xu. Atomistic molecular simulations of structure and dynamics of crosslinked epoxy resin. POLYMER. (2007). 48(19), 5802-5812.

[27] S.L. Mayo, B.D. Olafson, W.A. Goddard. Dreiding - a Generic Force-Field for Molecular Simulations. J PHYS CHEM-US. (1990). 94: 8897-8909.

[28] A.K. Rappé, C.J. Casewit, K.S. Colwell, W.A. Goddard, W.M. Skiff. UFF, a Full Periodic Tabel Force Field for Molecular Mechanics and Molecular Dynamics Simulations, J AM CHEM SOC. (1992) 114: 10024-10035.

[29] D.A. Vajari, C. González, J.Llorca, B.N. Legarth. A numerical study of the influence of microvoids in the transverse mechanical response of unidirectional composites. Compos. Sci. Technol. (2014) 97: 46-54.

[30] Huntsman Corporation. Datasheet. Araldite ${ }^{\circledR}$ LY 1556 / Aradur $^{\circledast} 917$ / Accelerator DY 070,http://www.huntsman.com/advanced_materials.

[31] B. Wetzel, P. Rosso, F. Haupert, K. Friedrich. Epoxy nanocomposites - fracture and toughening mechanisms. ENG FRACT MECH. (2006). 73(16): 2375-2398.

[32] T. Adachi, M. Osaki, W. Araki, S.C. Kwon. Fracture toughness of nano- and micro-spherical silica-particle-filled epoxy composites. ACTA MATER. (2008). 56: 2101-2109. 